
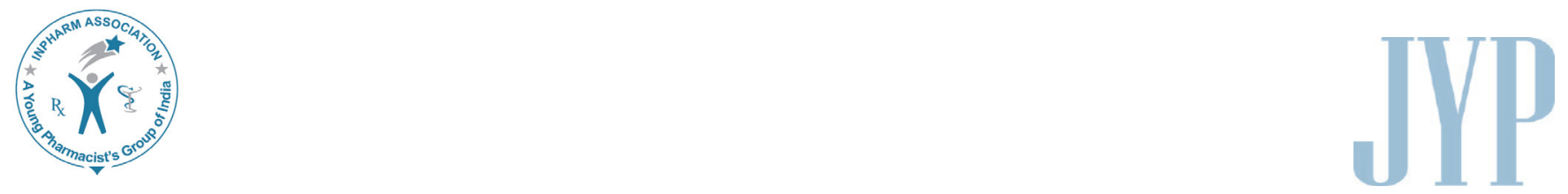

\title{
The Peripheral Focus on the Psychological Parameters of Parents of Autistic Children in the Intervention Methods: A Review and Recommending the Strategy, Focusing Psychological Parameters of Parents of Autistic Children in Intervention Methods
}

\author{
Vidya Bhagat", Nordin Bin Simbak and Mainul Haque \\ Faculty of Medicine, Universiti Sultan Zainal Abidin (UniSZA), 20400 Kuala \\ Terengganu, Terengganu, Malaysia.
}

\section{ABSTRACT}

The experience of parents with an Autism Spectrum Disorders (ASD) can be devastating. Parents and families have to cope with the compound, demanding situation in their life. The presence of pervasive and severe deficits in children with ASD increases the adjusting demands of parent's in their life situations. Those coping with life complexity with the parents of ASD nudge them into stress later into distress slowly incapacitates them that of their efficiency to deal with this situation. These parents are found with disturbances in their psychological parameters such as social, sexual, economic, and emotional. Perhaps this shatters them from their interpersonal relationship and family life. Indeed, these aspects of parental distress stand lower in position, and the focus goes with the treatment of ASD. Thus, the management of ASD by these parents to their deficit child capacitating to reach their fullest abilities remains questionable. Thus, there is a need for intervention of autistic children with a peripheral focus on psychological parameters of parents of ASD. This review study focuses on division of attention required for the treatment of ASD towards the child with autism and the parents who manage them.

Key words: Autism spectrum disorders, Emotional, Economic, Psychological mindedness, Social sexual.

\section{INTRODUCTION}

The ASD is a neurological and developmental disorder that is characterized by persistent impairment in shared

\begin{tabular}{|c|c|}
\hline \multicolumn{2}{|c|}{ Access this article online } \\
\hline Journal Sponsor & \multirow[b]{2}{*}{$\begin{array}{l}\text { Website: } \\
\text { www.jyoungpharm.org }\end{array}$} \\
\hline \multirow{2}{*}{ www.phcog net } & \\
\hline & $\begin{array}{l}\text { DOI: } \\
\text { 10.5530/jyp.2015.4s.1 }\end{array}$ \\
\hline
\end{tabular}

social communication and collaboration, repetitive forms of stereotype curiosity and behavioral accomplishments. These patterned symptoms are pervasive in nature manifest of early childhood in autistic children and limit their everyday functioning and affecting these children throughout their life. Diagnosis of ASD isn't only affecting the diagnosed child indeed, it affects the whole social system of the child. In one review study on analysis of the prevalence of Autism spectrum disorder in Asia, it is found that ASD was "probably more prevalent in Asia than

\footnotetext{
*Address for correspondence:

Prof. Vidya Bhagat, Professor, Unit of Psychiatry, Faculty of Medicine (FP), Universiti Sultan Zainal Abidin (UniSZA), 20400 Kuala

Terengganu, Terengganu, Malaysia.E-mail: 55vidya42@gmail.com
} 


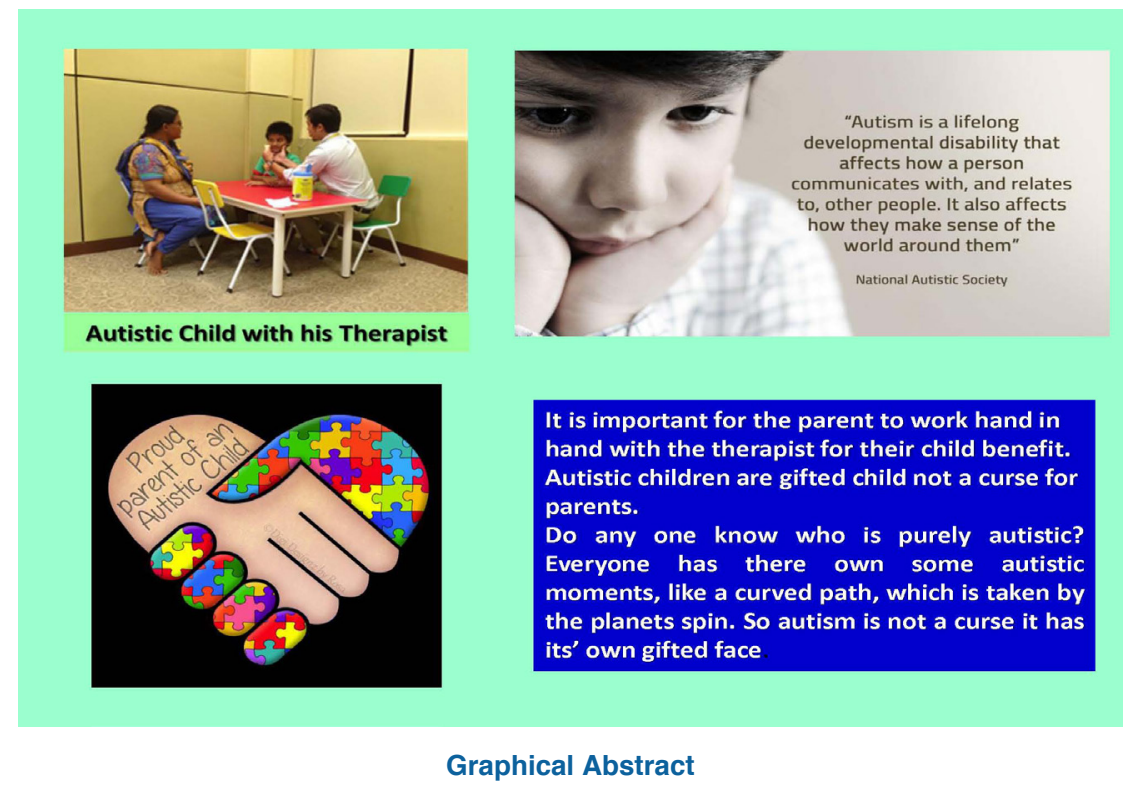

previously thought". The current the US prevalence rates of ASD are about 1 in 68 children. ${ }^{1}$ The rate of diagnosis seen towards ASD are increasing throughout the world. ${ }^{1}$

The stage at which functional impairment becomes evident will vary according to characteristics of each child with ASD and also depends on their environment. Core diagnostic features are visible in the developmental period, but intervention, compensation, and current supports and difficulties are disguised at least in some of the contexts. Manifestations of ASDs vary significantly in a severity of the autistic condition, developmental level, and chronological age. Thus, it is known to be the spectrum disorder. It occurs in all ethnic and socioeconomic groups and affects every age group. The experts is that 1 out of 88 children age 8 will have an ASD. ${ }^{2}$ The overall reported prevalence of ASD in recent studies was higher than the previously reported in Asia. ${ }^{3}$ The average prevalence of ASD before 1980 was around 1.9/10,000 while it was 14.8/10,000 from 1980 to present. The median prevalence of ASD among 2-6-yearold children who are reported in China from 2000 upwards was $10.3 / 10,000 .{ }^{3} \mathrm{ASD}$ is probably more common in Asia than previously thought. ${ }^{3}$ Males are four times more likely to have an ASD than females. ${ }^{3}$ Presently many research studies have been enthusiastically working for to grow with a better understanding of the biological roots of etiological factors in the manifestation of ASD. However, research on interventions intending to help ASD child and keeping their families with their psychological parameters in focus has found comparatively limited. Further, some literature demonstrates the evidence upon which service providers, family members, and researchers could make decisions about model adoption, selection for a family member, or future research. ${ }^{4}$
One of the added limitations here that the psychological stability of household members who are provided with the information and involving them in the decision-making process that often takes a crucial role in the intervention process. These parents who are the part of this intervention processes need to have psychological mindedness to involve them efficiently in this decision-making process. This review will focus on the four essential psychological parameters. Those are social, sexual, economic and emotional impact on psychological mindedness of the parents with intentional intervention for the whole family. These psychological parameters have their role in psychological mindedness and optimal functioning of these parents of ASD indeed getting involved in the decision-making process with interest and contentment.

\section{The psychological mindedness of the patents of ASD}

The Psychological mindedness of the parents with ASD children gets affected since they need to adapt to the demands of life because of their deficit child and related issues involved in psychological parameters. These psychological parameters grounded on social, sexual, economic, and emotional impacting on the mental status of parents with ASD children. The psychological mindedness's of these parents of the child with ASD seen with disturbances in these psychological parameters lodge various levels of stress on the parents which has an extended effect on understanding their children. It was reported that certain parental dimensions of stress are more with parents with ASD than that of other children with idiopathic developmental delay and usual development. ${ }^{5}$ Parenting stress is one of the frequently researched aspects perhaps most, of these researchers 
goes with clinical assessment of stress in acknowledging differences in parenting experiences in the parents of children with ASD. The evaluation of parental pressure is can be done by focusing on psychological parameters. Therefore the appropriate intervention program can be developed.

\section{Psychological parameters}

Social parameter

The dimensions of stress involve one or more of psychological parameters spelled earlier. One such is a social setting that has been focused in this review article that brings insightful views about parental stress seen as formed an association with disturbances in psychological mindedness. Raising and supporting a child with an ASD appears to hurt parents and families, regardless of a severity of symptomatology since the time of diagnosis. ${ }^{6}$ This review examines the results of "research in ASD according to the views of researcher's study the family hardiness is an important construct to understand coping in parents of individuals with ASD, who are regularly facing threat of substantial misery. This study examined family hardiness, perceived social support and parent self-efficacy as predictors of family distress in 138 mothers of individuals with ASD, 4-41 years of age. Using a multiple mediation analysis, we demonstrated that perceived self-efficacy and social support mediated the link between the pile-up of stressors and family hardiness, and that hardiness was a partial mediator in explaining how stressors were associated with family distress. Researchers and clinicians should consider the role that perceived social support and parent self-efficacy play in defining family hardiness as a psychological parameter. ${ }^{7}$ One study used structural equation modeling to examine the relationship between multiple sources of social care, hopefulness, and comfort of mothers of children with ASD. ${ }^{7}$

Social upkeeping was studied as a facilitator and arbitrator of the optimism-maternal safety. Moreover, the role of positivity as a umpire of the social support-maternal wellbeing association was also evaluated. Results discovered that family support was associated with increased confidence that, in turn, predicted higher levels of active maternal upshots and lower levels of adverse maternal aftermaths. Also, partner and friend support were directly associated with maternal consequences. Implications for the development of interventions directed at increasing the quality of social support networks are discussed. This review shows the significance of social parameter concerning parental psychological mindedness. ${ }^{8}$
Raising a child with an ASD presents significant challenges for parents that potentially have an impact on their health and well being. Further get more on this issue, the current study has inspected the extent to which parents experience exhaustion and its relationship to other aspects of prosperity and parenting. Mothers with ASD were suffering from more fatigue when compared with mothers of normal children. Features associated with high levels of weariness were poor maternal sleep quality, a great need for social support and poor quality of physical activity. Thus, in the social parameter of parents of ASD, social and family support gained by these parents becomes a bonus for intervening child with ASD. ${ }^{9}$

Therefore, this review study emphasizes the behaviors of a parent regarding the parenting stress and parameters that induce them, which becomes one of the impacting factors in the ASD child's behavior problem. The illustrating such views is examined in following a review study that see the sights the relationship between parenting activities in parents of children with Autistic Spectrum Conditions and subsequent child behavior complications. The sample consisted of 72 children and their parents, who were assessed over a period of 9-10 months. There was a relationship between parenting behaviors and subsequent child behavior problems, but only for the parenting behavior of limit setting. The better the limit setting of the parents at baseline, the fewer child behavior problems were noted at follow-up. Finally, the parenting behavior of limit setting was found to mediate the relationship between parenting stress and subsequent child behavior problems. ${ }^{10}$

Thus, in this focused area of current review probe into the psychological parameters which induce stress in the parents of ASD which indeed affect their psychological mindedness and also strains their parenting role and active involvement in intervention program of their deficit children. Parents of individual with ASD often experience stressors and distress associated with caring for their child. Approximately $0.9 \%$ of the population is thought to have an $\mathrm{ASD}^{11}$ and share a common triad in impairment of communication, socialization, behavior, and interests. ${ }^{12}$

Sexual Parameter

Carrying on with normal and happy family life is always questioned in parents of ASD. Marital issues disturbing psychological well-being are common in most of the marriages, but the extent is greater in a child with disabilities. Thus, marital dissatisfaction is one of the distressing issues among the parents of ASD. Examining the following reviews on marital satisfaction were lower in parents with ASD in the survey of parents/caregivers of a child 
with an ASD was conducted to examine the relationship between ASD characteristics, family functioning and coping strategies. A child with ASD places considerable stress on the family. Primary caregivers of a child with ASD from rural area of Australia $(\mathrm{N}=53)$ were surveyed for family functioning, marital satisfaction, self-esteem and coping strategies. Results suggest that these caregivers had healthy self-esteem, although they reported somewhat lower marital happiness, family cohesion, and family adaptability than did norm groups. ${ }^{13}$

Indeed, it has been examined that the marital dissatisfaction further leading to separation of parents with an autistic child is common among the parents of ASD. One of such study reviewed in the Journal of Family Psychology states that parents of autistic children had a $9.7 \%$ chance of getting divorced than did their peers. ${ }^{14}$ Marital stressors seen in these parents of ASD can include the following: (4 ways) $)^{14}$ Parents often admit their child's autism diagnosis at different times and in different ways, which causes struggle. Spending time together becomes difficult because of the numerous commitments and inconsistent schedules. It's often challenging to find child care for autistic children. Financial constant worry may cause hitches between partners. It has been reviewed that most parents of children with autism are acutely aware of the issues that come with the diagnosis and mainly, how stress affects their marriage. So what does the research say about autism and divorce? One study showed that parents who had a child with autism had a higher divorce rate than parents without a child with autism. ${ }^{15}$ However, a more recent study presented found that there was no upsurge in break up. ${ }^{16}$ This research found that " $64 \%$ of children with autism lived with married or adoptive parents, compared to a rate of $65 \%$ for children with no autism diagnosis. ${ }^{15}$

This issue of marriage is further examined to see the contribution of autism. Autism is not an alone leading cause in marital separations. However, most of the time autism marital dissatisfaction starts and ends with divorce. ${ }^{17}$ One of the supporting studies has been reviewed revealed that $78 \%$ of respondents said that they were divorced after their child was diagnosed, and overwhelmingly $76 \%$ of the respondents said that autism was not the primary cause of their divorce. Although the majority of respondents did not consider autism a leading cause of divorce, $50 \%$ did consider autism to be a contributing factor in the divorce. ${ }^{17}$ These reviewed studies that autism leading to parent's marital stress but also siblings are suffering from the whole issue. Review analyzes the views of the counselor should watch for high levels of marital stress that is reported to negatively affect typically developing sibling relationships with the child with autism. ${ }^{18}$
In general when there are issues like social, economic, and emotions aspects of an individual comes into marital pictures there will be some differences comes in all marriage commonly affect their sexual life. Since these issues are more frequent in the parents of ASD, indeed they face more concerned with their sexual life. These leads to suppressions and frustrations further affect their psychological wellbeing of parents of these children. Current work observed difficulties experienced by parents of children with autism in their sexual life. Another study ${ }^{19}$ used the method of focus group interviews and face-to-face in-depth interviews for qualitative research. Discussions were held with the parents of 12 autistic children registered at the Application and Research Center for a child with the disability shown that the parents expressed their unwillingness for any voice recording especially about sensitive issues. It was found that the parents of autistic children experienced difficulties in their family relations, social and economic spheres, and in coping with the education, and also the problematic behavior of their children and in their sexual lives. It was observed that a large part of the responsibility for giving care to their children was assumed by women and their interaction and sexual relationships with their husbands diminished for this reason. In view of these findings, the current study suggests that the nurses working in this particular field should provide professional social support to the parents and guide them to face the difficulties including their sexual life. Nurses also should inform the parents how and where they can obtain services in their critical need. ${ }^{19}$

\section{Economical parameter}

Among the psychological parameters, economic problems of parents of ASD are one of the significant which distress the parents and further disturbs their psychological mindedness and emotional well-being. Thus, self-blaming for giving birth to the child with the deficit and being the reason for the present condition is common with these parents. The current study overviews the statistical study on the review of the literature published from 1989 to 2005 for articles that examined the economic burden incurred by families as a result of caring for a child with disabilities. The review was performed according to a comprehensive economic conceptual model developed by the authors and to the guidelines set out by the Canadian Coordinating Office for Health Technology Assessment. An observation of these reviews in Canada, more than half a million children and people younger than 20 years old have some form of disability. Approximately one hundred fifty-five thousand children from 5 to 14 years of age live with activity limitations; of these, $43 \%$ live with severe to very severe limitations. ${ }^{20}$ 
One study estimates that autism costs the country at least f32 billion per year in treatment, lost earnings, care and support for children and adults with autism. This study also estimates more than 600,000 people in the UK have autism, a condition associated with poor social and communication skills and restricted, repetitive patterns of behavior. A quarter of people with autism are unable to talk, and $85 \%$ do not work full time. ${ }^{21}$

Further present review research makes an effort to analyze these aspects, reviewing the research in this area. Reviewing one of such study had investigated how child characteristics influence maternal parenting stress and psychological distress. Participants consisted of mothers and developmental age-matched preschool-aged children with ASD ( $\mathrm{N}=51)$ and Developmental disorders (DD) without autism $(\mathrm{N}=22)$. Evidence for higher levels of parenting stress and psychological distress was found in mothers in the ASD group compared to the DD group. Children's problem behavior was associated with increased parenting stress and psychological distress in mothers in the ASD and DD groups. Perceived parental competence and marital satisfaction were lower in parents of children with ASDs further self-blame and disruption of finances and activities are found equivalent to the children with developmental disorder. ${ }^{22}$

This review research examined the stress due to burning out the pockets of ASD parents that need to be sighted in. Child care and burdening the family income revealed in one of the studies examined here is the parents of children with autism have significant out-of-pocket expenditures related to their child's care. ${ }^{23}$ The impact of having a child with autism on household income is not known. Has been reviewed which has been revealed the fact of financial burn outs in the parents' ASD child. This study had estimated the loss of household income associated with childhood autism using a nationally representative sample. An ASD was defined as an affirmative response to the questions, "has a health professional. There were 131 children with the ASD. One study reported that having a child with autism spectrum disorder were associated with decreased odds of living in a higher income household after controlling for parental education, type of family, parental age, location of the home, and minority ethnicity. The average loss of annual income associated with having a child with autism spectrum disorder was $\$ 6200$ or $14 \%$ of their reported income. ${ }^{23}$ This study had theorized that childhood autism is associated with a substantial loss of annual household income. This likely places a significant burden on families in the face of additional out-of-pocket expenditures.
The emotionality of parents of ASD child is also the most important psychological parameter that has been affected by all other parameters. The financial aspect disturbing emotional spectrum is examined by the researcher to see the impact on one another. One of the supporting review citing examples here is a study that describes the effects of Caregiving for parents of children with ASD. Secondly, we investigate, construct validation of the care-related quality of life instrument measuring the impact of Caregiving. Primary caregivers of children with ASDs were included. Many parents have experienced considerable problems combining daily activities with care, had financial problems or suffered from the depressive mood. Validity tests showed that a higher impact of caring on the CarerQol was positively associated with higher subjective burden and lower family quality of life. ${ }^{24}$

\section{Emotional parameter}

Parent's emotionality is an important parameter changing psychological mindedness to work with their child with ASD and their full involvement in the intervention program. Disturbances in this setting may reduce their efficiency with this regard. This review study examines the related aspect depicted in the study in which fatigue was also significantly related to other aspects of wellbeing, including stress, anxiety and depression, and lower parenting efficacy and satisfaction. The need for interventions to specifically target parental fatigue and its impact on families affected by ASDs both in the short and long term is clearly indicated. ${ }^{25}$

Parents of children with developmental disabilities, particularly with autism spectrum disorder, are at risk for high levels of distress. Further the current study focuses on parents of ASD children, their emotions and mental stability to understand the psychological parameter. One of such area saw in which the cognitive component is the disturbing effect in different angles on parents of $\mathrm{ASD}^{22}$ Parents of children with ASD typically report higher levels of parenting stress and greater affective symptoms when compared with normal and other disabilities. ${ }^{26}$ Ultimately, to have a better understanding of these relationships to focus on the formulation of modified interventions and improve the scope of studies evaluating the outcome of therapies for ASD.

One of the examined reviews reveals that the relationships between parenting stress and self-perceived parenting behaviors in 138 parents of children with autistic spectrum conditions were studied over 9-10 months. Parenting stress closely interacted with self-perceived involvement, communication, and limit setting over time. Parents of young children (below 4), high initial levels of 
parenting stress resulted in less subsequent self-perceived involvement, and poorer communication, with the child. Good self-perceived first skills for limit setting resulted in lower levels of parenting stress. These relationships help to explain the impact of parenting stress on child behavior problems, and may be consistent with the development of parental adaptive behavioral strategies to deal with extreme stress levels. ${ }^{27}$ After viewing the study results of emotional stress in mothers of 110 children with ASD were interviewed with the Child and Adolescent Impact Assessment when their children were approximately nine years old. Regression analyzes revealed that African American mothers reported lower levels of perceived negative impact of having a child with ASD than did Caucasian parents. Higher repetitive behavior scores on the Autism Diagnostic Interview-Revised, lower adaptive behavior scores on the Vineland Adaptive Behavior Scales, and less perceived social support were also significant predictors of higher perceived negative impact. Identifying predictors of perceived negative impact are an important first step in designing interventions to support families and target parents who may be at risk for experiencing higher levels of stress. ${ }^{28}$

In an exemplary study of comparison made between the developmental disorder children with their parental characteristics revealed that autistic boys aged 5 to 10 years and without evidence of either mental retardation or neurological disease. Measures were devised specifically to assess those parental characteristics and environmental stresses hypothesized to play a part in the genesis of autism. The autistic group contained a larger proportion of middle-class families. No differences between the groups were found with respect to parental warmth, emotional demonstrativeness/responsiveness, or sociability, parental psychiatric disorder, or with respect to early stresses of any kind. It is concluded that it is unlikely that autism develops as a consequence of such parental personality attributes. ${ }^{29}$

This review further examines the possibilities of gaining more information about the supporting system in the studied article that presents a grounded theory analysis of the messages sent to an email group by parents of children with autism. The core category emerging from the study indicates that the group functioned in making sense of autism. The core group comprises four categories: searching for meaning; adjusting to changes; providing support and encouragement; and narrative sharing of experiences. Although email groups function as a social support mechanism, we suggest that this is not the only legitimate purpose: such groups may contribute towards the development of parental representations of autism. ${ }^{30}$
One of the examined research studies depicts the picture of the parental emotional expression of a child with ASD. In this study the performance on an emotional labeling task in response to schematic facial patterns representing five basic emotions without the concurrent presentation of a verbal category. 'Autism fathers' performed worse than mothers that performed worse than controls in decoding displays representing sadness or disgust. This study also opens up a need to include facial expression decoding tasks in genetic research of autism. Also, emotional expression interactions between parents and their children with autism, particularly through play, where affect and prosody are 'physiologically' exaggerated, may stimulate the development of social competence. $^{31}$

Self-efficacy has been known to be an important variable affecting parent outcomes in the management of children with ASD. Review examining in one of such study on 26 mothers and 20 fathers of children with autism reported on their self-efficacy, anxiety, and depression. In which the regression analyzes showed that self-efficacy mediated the effect of child behavior problems on mothers' anxiety and depression, but there was no evidence that it functioned as a mediator for fathers. However, there was evidence that self-efficacy moderated the effect of child behavior problems on fathers' anxiety. ${ }^{32}$

A present review study has refereed associations between parenting a child with autism and stress indicators. Responses were compiled in the Aggravation in Parenting Scale. Parents of children reported to have autism (N=459) were compared with parents of i. children with special health care needs, including emotional, developmental, or behavioral problems other than autism that necessitated treatment (children with other developmental problems ( $\mathrm{N}=4545)$; ii. Children with special health care needs without developmental problems ( $\mathrm{N}=11475)$; and iii. Children without special health care needs $(\mathrm{N}=61826)$. Weighted estimates are presented. ${ }^{33}$

Parents of children with autism were more likely to score in the high aggravation range (55\%) than parents of children with developmental problems. However, within the autism group, the proportion of parents with great annoyance was $66 \%$ for those whose children recently needed special services and $28 \%$ for those whose children did not. The parents of children with autism and recent special service needs were substantially more likely to have a high aggravation than parents of children with current individual service requirements in each of the three comparison groups. Conversely, parents of children with autism, but without current individual service needs were 
not more likely to have a high aggravation than parents of children with other developmental problems. ${ }^{33}$

A cross-sectional comparison of child behavioral and emotional problems and maternal mental health measures among 18,415 children (5-16 years). Among them, 47 had an ASD, 51 combined ASD with ID, 590 had only ID, and the remainder was the comparison group with no ASD or ID. The result here revealed that ASD and ID are independent risk factors for behavioral and emotional problems. ASD (but not ID) is positively associated with the maternal psychological disorder. Approaches to diagnosing hyperactivity and conduct problems in children with ASD may need to be reconsidered. ${ }^{34}$

Parents of children with the autism were found higher rates of alcoholism. Examination of past literature reviews had revealed the family histories of alcoholism and other addictive disorders, often not focused on in the medical genetics clinic, should be ascertained. In a study of 36 autism families, study also compared the lifetime rates of psychopathology. The study was based on direct SADS-LA interviews of parents and adult siblings of autistic children. It was reported that $47 \%$ $(17 / 36)$ of the autism families had a first-degree relative with substance abuse, including alcoholism, versus none in the 21 control families. Also, 22\% of first-degree relatives reported substance abuse compared with none in the controls $(\mathrm{P}=0.002)$. They also found increased rates of depression $(32.3 \%$ vs. $11.1 \% ; \mathrm{P}=0.013)$ and social phobia $(20.2 \%$ vs. $2.4 \%$; $\mathrm{P}=0.016)$. It was reported pedigree analyses of 167 autism families finding that $39 \%$ of autism-ascertained families could be classified as having probable genetic alcoholism. ${ }^{35}$

One of the study focused on the coping strategies of parents' with children with Autistic spectrum disorders (ASD) and the relation between these strategies and parenting styles. Two groups of participants were included: parents with children with ASD (EG) (n=66) and a matched control group $(\mathrm{CG})(\mathrm{n}=66)$. Paired Samples t-test and Pearson's r correlation were used as methods of analysis. Main results distinguished significant $(p<0.001$ to 0.003 ) differences between the EG and CG for almost all variables included. The $\mathrm{M}$ level of coping strategy was much higher for the CG than for the EG. SOC showed a stress-reducing effect in both the EG and CG. PIL-R explained $50 \%$ of the variance in SOC for the EG and 33 $\%$ for the CG. The only significant gender difference in the EG was on SOC indicating a higher sense of coherence among the fathers and probably an indicator of a stronger burnout effect of the mothers. ${ }^{36}$

\section{CONCLUSION AND INTEGRATION}

A New Model for ASD intervention program recommending considering the peripheral focus on the psychological parameters disturbing psychological mindedness of the parents with autistic children. On this base, this research reviewed the points above and suggested that i) ASD are severe, persistent and dynamic. ii) These deficits are associated with plentiful difficulties of parents with ASD in in the psychological parameters such as social, sexual, economic and emotional. iii) The impact of ASD on parents possible to have a reciprocal negative effect on children with ASD, perhaps this adverse impact could ultimately lessen positive effects of the intervention. iv) There are wide ranges of responses open for children with ASD, even with parent and family involvement, which is most important in effective therapy. v) Evaluation of ASD therapies is often limited to assessment of the child, without consideration of the psychological mindedness of parents who are the part of treatment.

In a review related to assessing subjective parameter in the interventions for ASD, had commented on the: Modification of existing measures and creation of new measures of outcome in autism should be a high priority in order to document the goals for and effects of treatment. The focused area in his study which extends distinct interest which had measured parent-child interaction, family satisfaction, adverse effects of treatment, and semi-structured observations or inquiries targeted areas of change identified by parents or teacher of individuals with autism. ${ }^{37}$

One of the studies addressed the need for the strategies with additional support. This study had addressed the daily challenges faced by parents of children diagnosed with the autism spectrum disorder. This article reports on a qualitative interview study with 20 parents exploring their experiences, challenges faced, and what has helped them to cope. A thematic analysis of the data identified five core categories: Dealing with challenging behavior; dealing with judgments from others; lack of support; impact upon the family; coping and the importance of appropriate support. The findings emphasize where the parents themselves believe they still require additional support. It raises key strategies and resources that parents have found helpful. Caring for children with ASDs is challenging and affects family life. ${ }^{38}$

Therefore, the classical peripherally focusing interventions on the parents with ASD child is suggested (Figure 1). This model is intended to be prescriptive in nature, encouraging 


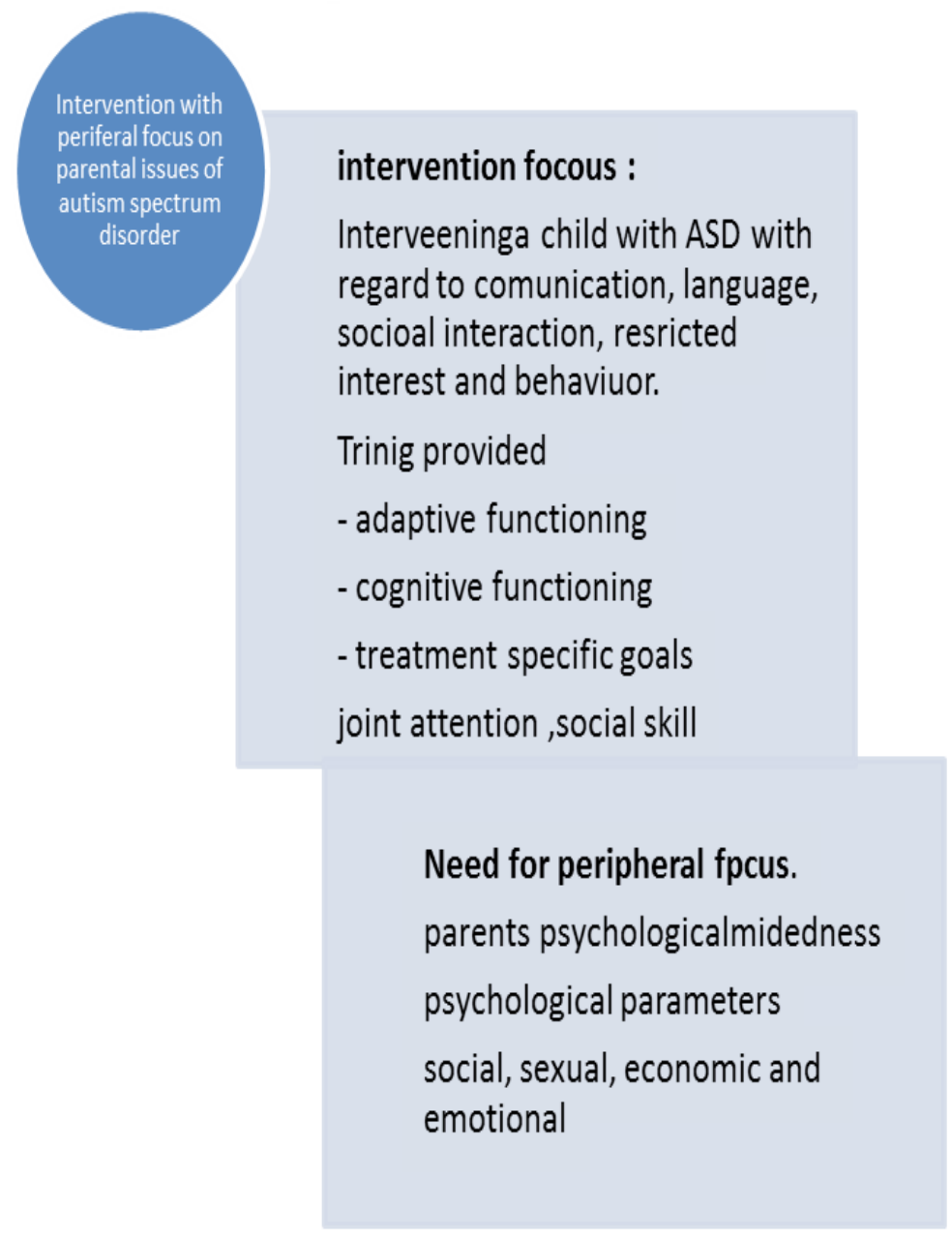

Figure 1: The peripheral focus in the intervention for ASD

those engaging in the ASD intervention and research to focus on psychological mindedness. The suggested intervening program focuses on four psychological parameters social, sexual, economic and emotional. The disturbances in this area have been verified by research to be connected. These four focused areas can be intervened to bring better off in the psychological mindedness. Here, peripheral intervention can be extended by hypnotherapy, cognitive hypnotherapy, yoga, individual psychotherapies and supportive programs by the government and other health preventing agencies strategized for the parents with ASD. This model of treatment evaluation thus follows but redistributes the five dimension type of treatment outcome evaluation which included symptoms and diagnosis, functioning, consumer perspectives, environments, and system (SFCES model) $\cdot^{39}$ In consideration of the SFCES framework, the proposed model assesses the symptoms and functioning of both child and parent. The overall operation of the family and the family environmental chaos, and the systemic interactions present among the child, siblings, and caregivers. ${ }^{39}$
The redistribution of variables into two primary domains helps clarify the transnational nature of the relationship between childhood ASD deficits and parent and family distress. While still capturing the "dynamic and interaction interplay between the child and his or her environments over time. ${ }^{40}$ Further, it borrows from the ecological model of human development as well as family systems theory in supposing that a change in any dimension of the family has an impact on other individuals and relationships. ${ }^{41-42}$

Ultimately, data amalgamated from studies using this model may be used to develop and test nuanced models describing the interplay between child, parent, and family variables. This will in turn will help facilitate more improved understanding of "effective ingredients" of therapy. ${ }^{43}$ At this time, however, just knowing more about how various ASD treatments impact parents and families are of primary necessity. The proposed model should not only be applied to modalities of intervention with direct parent and family involvement. In fact, many therapy models incorporating caregivers, such as parent training models, have included 
some evaluation of parental outcomes as they are the direct receiver of treatment in these instances. The greatest "gap" in current ASD intervention research is within therapeutic contexts that do not directly involved, but no doubt affect, parent, and family functioning. This omission has resulted in incomplete understanding of therapeutic impact as well as factors contributing to the long-term maintenance or sustained growth following treatment. The fanatical parameter need to be taken into consideration that may be a peripheral focus. Nonetheless this area is found to be an impacting role in distressing part and hampering the treatment outcome further research is recommended focusing on this parameter. Equity relates to fairness, in the sense that the economic burden may be too high for some. Equity refers to income, and lack there off has been recognized by the WHO as an important determinant of ill health. ${ }^{20}$

Another factor recommended to focus in the intervention, and further research is emotionality of the parents of a child with ASD. Assessing the level of distress in these parents and helping them psychologically with peripheral interference. Further, collecting the data of the benefits of the upcoming research may bring more insight with this regard. It is an example study concerning this variable stress; anxiety and depression are raised amongst parents of children with a developmental disorder. However, the processes by which stress leads to depression and anxiety are poorly understood. A cross-sectional survey assessed levels of parental stress, depression and anxiety among parents of children with autistic disorder, children with Down's syndrome and children with no disorder. Anxiety and depression were highest in parents of children with autism. Locus of control was more external in parents of children with autism. ${ }^{44}$ The recommended strategy includes the (A) evaluating psychological parameter that should include assessment, social, sexual, economic and emotional domain of parents prior to getting involved in the therapeutic intervention of their child. (B) Psychologically intervening the related parameters and strengthening their psychological mindedness. It is imperious that examination of intervention strategies of ASD reporting parental psychological parameters, otherwise, it may hamper the scope by ignoring the context induced by the peripheral aspects, in which children develop and in which ASD interventions are set and continued in its application.

Finally, parental psychological mindedness should be focused peripherally in the treatment and should be considered an indicator of the overall benefits of therapy, along with measures taken for the child's benefit. This strategy would help the clinicians to determine whether parents had benefitted from treatment peripherally provided to them and its overall impact on child's treatment. Further, this approach could serve as an indicator of the parent's readiness to utilize techniques suggested during treatment and ability to continue finding appropriate support and intervention for their child. Ultimately, the incorporation of this treatment strategy provides the clinician with a way to assess the overall functioning of the parents of these children from the beginning of treatment to the end. Further to hold control over regular planned follow-up visits after the completion of an intervention program. In the case of revenues to treatment, citing a plateau in improvements that had been made, prior assessment of variables in the psychological parameter will help the clinician to more speedily find the area of concern, to understand factors that are likely obstructing the progress, and make recommendations for the appropriate changes needed.

Further, use of this strategy will provide greater insight into the effectiveness of ASD treatments. Longitudinal research throughout treatment may help develop better understanding of the components of treatment that are most meaningful or beneficial to parents and families, to gain better therapeutic benefit. ${ }^{45}$ The intensive nature of most ASD interventions suggests a need for better understanding of the mechanisms of treatment which brings the change for the betterment. Thus, the recommended strategy will facilitate to boosting of the understanding regarding the psychological parameters are focused on the intervention and helping the child with ASD by the peripheral attention given to bring the psychological mindedness among the parents of these children thereby improving their cognitive efficacy and peeling of distressing parameters that occur throughout therapy. Also, incorporating parent into the assessment of ASD intervention should help to understand what factors promote continued child improvements all over the treatment and also in the later part. However, long-term treatment follow-up research is often restricted by incomplete understanding of therapeutic benefits and the parameters involved in limiting the advantages and the difficulties in overcoming the complexities of the intervention and related research. Same study ${ }^{45}$ recommended for improving the understanding of how changes are maintained in assessment treatment outcomes. Incorporation of parental factors into therapy outcome research will help fill the negated area in the treatment and the follow-ups, giving context to the environment that the child with ASD has been involved in the intervention. The suggested response can be combined with a variety of treatment interventions, but would be best employing the longitudinal intervention research with assessment of the 
outcome benefits before, during, and immediately after the intervention and at the face of long-term follow-ups. In addition to assessing for statistical significance and effect size, these parameters should be evaluated with respect to a clinically meaningful change to help fully understand the practical benefits of interventions to parents. ${ }^{46}$ The significance of parental role is so significant thus parents' societies have traditionally been very important to the field of autism. The first autism societies in the US and UK many years ago. The current research support and advocacy provided by a number of parent-founded, parent-led, and parent-supported organizations. These groups have already begun to be influential in terms of research, especially in funding new investigators and attracting eminent scientists from other fields into studying the disorder. The parents' organizations also have the potential to be extremely helpful in the dissemination of scientific information, particularly on the Internet. ${ }^{47}$ Thus, strategies introduced psychological mindedness is recommended which would help parental society in this regard.

Further, the variables suggested in this model should be assessed for both main and interaction effects to finest confinement and understand the multifaceted and symbiotic relationship between the paradigms. As a final point, additional variables can and should be added if it is apt to the therapeutic benefits. If similar outcome measures are utilized, this would eventually allow for more comprehensive comparison between ASD treatments, developing better-coping skills and work more mindfully in the provided intervention to get the possible benefits. The concept of psychological mindedness originated in the psychoanalytic literature; its use has been expanding beyond the realm of assessing suitability for, or psychic change following, psychotherapy or psychoanalysis. ${ }^{48}$

The complexity of analyzing psychological aspects and helping the parents to gain psychological mindedness along with the primary task in the treatment of ASD is not an easy task. Nevertheless the jeopardy of not doing so when evaluating the effectiveness of ASD interventions far overshadows the flagrancy of the task required. However, mindfulness in parenting may have benefits for parents' psychological health. Further research exploring the effectiveness of mindfulness in parenting interventions is warranted. ${ }^{49}$ The studies under review collectively suggest that the practice of mindfulness in everyday life over significant periods of time can both improve the experience of care providers. ${ }^{50}$ Furthermore, with limitation the clinicians workouts, and more upcoming researches with this regard are beneficial in the interventions of ASD.

\section{ACKNOWLEDGEMENT}

Authors are grateful to Universiti Sultan Zainal Abidin for all cooperation.

\section{CONFLICT OF INTEREST}

This study obtain no funding. Authors possesses no conflict of interest.

\section{ABBREVIATION}

ASD: Autism Spectrum Disorders

\section{Highlights of Paper}

- The experience of parents with an Autism Spectrum Disorders (ASD) can be devastating.

- The presence of pervasive and severe deficits in children with ASD increases the adjusting demands of parent's in their life situations.

- Coping with life complexity with the parents of ASD nudge them into stress later into distress slowly incapacitates them that of their efficiency to deal with this situation.

\section{Author Profile}

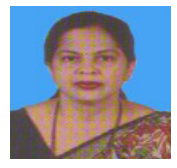

- Dr. Vidya Bhagat: Is a Professor of Unit of Psychological Medicine, Faculty of Medicine, UniSZA. Dr Bhagat has teaching experience of Psychiatry and Behavioural Science for under and postgraduate medical and paramedical students for last 22 years in different countries. Her chief area of interest is working on negative emotionality, which is a personality dimension. Her research interest also extended towards the intervention of autistic children and parenting. 


\section{REFERENCES}

1. Lord C, Bishop SL. Autism Spectrum Disorders Diagnosis, Prevalence, and Services for Children and Families Social Policy Report, sharing child and youth development knowledge. Soc Policy Rep. 2010; 24(2): 1-27.

2. Centers for Disease Control and Prevention. U.S. Department of Health and Human Services. Prevalence of Autism Spectrum Disorders-Autism and Developmental Disabilities. MMWR. 2012; 61(3): 1-18.

3. Sun X, Allison C. A review of the prevalence of Autism Spectrum Disorder in Asia. Res Autism Spectr Disord. 2009; 4(2): 156-67.

4. Odom SL, Boyd BA, Hall LJ, Hume K. Evaluation of Comprehensive Treatment Models for Individuals with Autism Spectrum Disorders. J Autism Dev Disord 2010; 40(4): 425-36.

5. Autism. Author manuscript. Parenting stress and psychological functioning among mothers of preschool children with autism and developmental delay. Autism 2009; 13(4): 375-87.

6. Pottie CG, Ingram KM. Daily stress, coping, and well-being in parents of children with autism: A multilevel modeling approach. J Fam Psychol. 2008; 22(6): 855-64.

7. Weiss JA, Robinson S, Fung S, Tint A, Chalmers P, Lunsky Y. Family hardiness, social support, and self-efficacy in mothers of individuals with Autism Spectrum Disorders. Res Autism Spectr Disord. 2013; 7(11): 1310-7.

8. Ekas NV, Lickenbrock DM, Whitman TL. Optimism, Social Support, and Well-Being in Mothers of Children with Autism Spectrum Disorder. J Autism Dev Disord. 2010; 40(10): 1274-84.

9. Still stressed but feeling better: Well-being in autism spectrum disorder families as children become adults. SAGE Journals. Autism 1362361315583191, first published on May 8, 2015 Psychology, Developmental 13 out of 68 .

10. Osborne LA, Mchugh L, Saunders J, Reed P. The effect of parenting behaviors on subsequent child behavior problems in Autistic Spectrum Conditions. Res Autism Spectr Disord. 2008; 2(2): 249-63.

11. Centers for Disease Control and Prevention (CDC). US Department of Health and Human Services. CDC estimates 1 in 68 children has been identified with autism spectrum disorder. 2014. Available in http://www.cdc.gov/media/releases/2014/p0327autism-spectrum-disorder.html

12. Weiss JA, Lunsky Y. The brief family distress scale: A measure of crisis in caregivers of individuals with autism spectrum disorders. J Child Fam Stud. 2011; 20(4): 521-8.

13. Higgins DJ, Bailey SR, Pearce JC. Factors associated with functioning style and coping strategies of families with a child with an autism spectrum disorder. Autism 2005; 9(2): 125-37.

14. Paltrow C. 4 Ways a Child with Autism Affects Family Life. Psych Central. Retrieved on July 7, 2015, from http://psychcentral.com/ blog/archives/2015/02/25/4-ways-a-child-with-autism-affectsfamily-life/

15. Freedman BH, Kalb LG, Zablotsky B, Stuart E. A Relationship Status among Parents of Children with Autism Spectrum Disorders: A Population-Based Study. J Autism Dev Disord. 2012; 42(4): 539-48.

16. Grosso K. Do Couples Divorce Because Of Autism? Psychology Today 2011; https://www.psychologytoday.com/blog/autism-in-reallife/201103/do-couples-divorce-because-autism

17. Department of Justice. High-Conflict Separation And Divorce: Options For Consideration 2004; http://www.justice.gc.ca/eng/ rp-pr/fl-If/divorce/2004_1/pdf/2004_1.pdf

18. Coogle CG, Guerette AR, Hanline MF. Early Intervention Experiences of Families of Children with an Autism Spectrum Disorder: A Qualitative Pilot Study. Early childhood research and practice 2013; 15(1)

19. Aylaz R, Yılmaz U, Polat S. Effect of Difficulties Experienced by Parents of Autistic Children on Their Sexual Life: A Qualitative Study. Sex Disabil. 2012; 30(4): 395-406.
20. Anderson D, Dumont S, Jacobs P, Azzaria L. The Personal Costs of Caring for a Child with a Disability: A Review of the Literature. Public Health Rep. 2007; 122(1): 3-16.

21. The London School of Economics and Political Science. Autism is the most costly medical condition in the UK. 2014 | http://www. Ise.ac.uk/newsAndMedia/news/archives/2014/06/Autism.aspx

22. Estes A, Munson J, Dawson G, Koehler E, XH Zhou, Abbott R. Parenting stress and psychological functioning among mothers of preschool children with autism and developmental delay. Autism 2009; 13(4): 375-87.

23. Montes G, Halterman JS. Association of childhood autism spectrum disorders and loss of family income. Pediatrics 2008; 121(4): e821-6.

24. Hoefman R, Payakachat N, Van Exel J, Kuhlthau K, Kovacs E, Pyne J, et al. Caring for a Child with Autism Spectrum Disorder and Parents' Quality of Life: J Autism Dev Disord. 2014; 44(8): 1933-45.

25. Hill C, Rose J. Parenting stress in mothers of adults with an intellectual disability: Parental cognitions in relation to child characteristics and family support. J Intellect Disabil Res. 2009; 53(12): 969-80.

26. Davis NO, Carter AS. Parenting stress in mothers and fathers of toddlers with autism spectrum disorders: associations with child characteristics. J Autism Dev Disord. 2008; 38(7):1278-91.

27. Osborne LA, Reed P. Stress and self-perceived parenting behaviors of parents of children with autistic spectrum conditions. Res Autism Spectr Disord. 2010; 4(3): 405-14.

28. Bishop SL, Richler L, Cain AC, Lord C. Predictors of perceived negative impact in mothers of children with autism spectrum disorder. Am J Ment Retard. 2007; 112(6s): 450-61.

29. Cox A, Rutter M, Newman S, Bartak L. A comparative study of infantile autism and specific developmental receptive language disorder II. Parental characteristics. BJ Psych. 1975; 126(2): 146-59. http://bjp.rcpsych.org/content/126/2/146.full-text.pdf+html

30. Huws JC, Jones RS, Ingledew D. Parents of children with autism using an email group: A grounded theory study. J Health Psychol. 2001; 6(5): 569-84.

31. Palermo MT, Pasqualetti P, Barbati G, Intelligente F, Rossini PM. Recognition of schematic facial displays of emotion in parents of children with autism. Autism 2006; 10(4): 353-64.

32. Hastings RP, Brown T. Behavior problems of children with autism, parental self-efficacy, and mental health. Am J Ment Retard. 2002; 107(3): 222-32.

33. Schieve LA, Blumberg SJ, Rice C, Visser SN, Boyle C. The relationship between autism and parenting stress. Pediatrics 2007; 119(Suppl 1): S114-21.

34. Totsika V, Hastings RP, Emerson E, Lancaster GA, Berridge DM. A population-based investigation of behavioural and emotional problems and maternal mental health: Associations with autism spectrum disorder and intellectual disability. J Child Psychol Psychiatry 2011; 52(1): 91-9.

35. Miles JH. Autism spectrum disorders-A genetics review. Genet Med. 2011; 13(4): 278-94

36. Sivberg B. Coping strategies and parental attitudes, a comparison of parents with children with autistic spectrum disorders and parents with non-autistic children. Int $\mathrm{J}$ of Circumpolar Health 2002; 61(Suppl 2): 36-50.

37. Begeer S, Gevers C, Clifford P, Verhoeve M, Kat K, Hoddenbach $\mathrm{E}$, et al. Theory of Mind Training in Children with Autism: A Randomized Controlled Trial. J Autism Dev Disord. 2011; 41(8): 997-1006.

38. Ludlow A, Skelly C, Rohleder P. Challenges faced by parents of children diagnosed with autism spectrum disorder. J Health Psychol. 2012; 17(5): 702-11.

39. Hoagwood KE, Jensen PS, Acri MC, Serene Olin S, Lewandowski R, Herman RJ. Outcome Domains in Child Mental Health Research 
Since 1996: Have They Changed and Why Does it Matter? J Am Acad Child Adolesc Psychiatry 2012; 51(12): 1241-60.e2.

40. Sameroff A. The transactional model of development: How children and contexts shape each other. American Psychological Association, Washington DC, USA; 2009.

41. Bronfenbrenner $U$. Recent advances in research on the ecology of human development. In: Development as action in context. Springer Berlin Heidelberg; 1986. 287-309.

42. Minuchin P. Families and individual development: Provocations from the field of family therapy. Child development 1985; 56(2): 289-302.

43. Littell JH. Manual-based programs for parents and children: How do we know what works? 2012. file:///C:/Users/UDM/Downloads/ Littell+WhatWorks+1June2012.pdf

44. Hamlyn-Wright S, Draghi-Lorenz R, Ellis J. Locus of control fails to mediate between stress and anxiety and depression in parents of children with a developmental disorder. Autism 2007; 11(6): 489-501.
45. Sheinkopf SJ, Siegel B. Home-based behavioral treatment of young children with autism. J Autism Dev Disord. 1998; 28(1): 15-23.

46. Jacobson NS, Truax P. Clinical significance: a statistical approach to defining meaningful change in psychotherapy research. $J$ Consult Clin Psychol. 1991; 59(1): 12-9.

47. Lord C, Cook EH, Leventhal BL, Amral DG. Autism spectrum disorders. Neuron 2000; 28(2): 355-63.

48. Shill MA, Lumley MA. The Psychological Mindedness Scale: factor structure, convergent validity and gender in a non-psychiatric sample. Psychol Psychother 2002; 7(Pt2): 131-50.

49. Beer M, Ward L, Moar K. The relationship between mindful parenting and distress in parents of children with an autism spectrum disorder. Mindfulness 2013; 4(2): 102-12.

50. Yoon-Suk H, Kearney P. Mindful and mutual care for individuals with developmental disabilities: a systematic literature review. J Child Fam Stud. 2014; 23(3): 497-509. 